\title{
SUPERLUMINAL GRAVITATIONAL MICROLENSING EVENTS IN PKS 0537-441 AND PROSPECTS FOR FUTURE DETECTION:
}

G.C. SURPI

Depto. de Física, FCEyN, Universidad de Buenos Aires Ciudad Univresistaria - Pab. I, 1428 Bs. As., Argentina

G.E. ROMERO

Instituto Argentino de Radioastronomía

CC5, 1894 Villa Elisa, Bs. As., Argentina

AND

H. VUCETICH

FCAyG - Universidad Nacional de La Plata

Paseo del Bosque S/N, 1900 La Plata, Bs. As., Argentina

\begin{abstract}
The BL-Lac object PKS 0537-441 has recently displayed strong intraday variability at $1.42 \mathrm{GHz}$ on time scales of $\sim 10^{4} \mathrm{~s}$. Such kind of variability is hardly consistent with an intrinsic-to-source origin, since apparent brightness temperatures higher than $10^{21} \mathrm{~K}$ are implied for the emitting region in the source.

An alternative explanation for this ultra-rapid radio variability in PKS 0537-441 is developed based on gravitational microlensing of a superluminal component in the blazar caused by compact objects in the halo of an intervening galaxy. The mass of the microlenses is estimated and the blazar is proposed as a good candidate in a systematic search for extragalactic MACHOs.
\end{abstract}

\section{The Model}

The underlying idea in our model, "superluminal microlensing", was originally proposed by Gopal-Krishna and Subramanian (1991). A foreground galaxy with $z=0.186$ and a close alignment with PKS 0537-441 was reported by Stickel et al. (1988). In the present case, the focusing can be 
approximated by (Romero et al. 1995):

$$
k=\frac{\Sigma_{\mathrm{c}}}{\Sigma_{c r i t}}=\frac{4 \pi G D_{\mathrm{d}} D_{\mathrm{ds}} \Sigma_{\mathrm{c}}}{c^{2} D_{\mathrm{s}}}
$$

where $\Sigma_{c}$ is the central surface density in the galaxy and the rest of the symbols have their usual meanings. During a microlensing event the total amplification of the background source is:

$$
A=1-\frac{1}{(1-k)^{2}}+\frac{2(1-k)+u^{2}}{(1-k)^{2} u \sqrt{4(1-k)+u^{2}}},
$$

where $u$ is the distance from the source to the microlens of mass $M$ in the lens plane, in units of the Einstein radius $R_{\mathrm{e}}=\left[4 G M D_{\mathrm{d}} D_{\mathrm{ds}} / c^{2} D_{\mathrm{s}}\right]^{1 / 2}$. If $u$ is expressed as a function of the relative source-lens trajectory, then equation (2) can be used to fit the observed variability reported by Romero et al. (1994), with $k$ restricted to the range $0.2 \leq k \leq 0.6$ imposed by the magnification of the unique macroimage of the blazar. From the results of these fits we estimate the microlenses have masses between $3 \times 10^{-5} M_{\odot}$ and $3 \times 10^{-3} M_{\odot}$ for superluminal velocities in the 1-10c range (in the lens plane). Additionally, from the event rate, we estimate a central dark mass density $0.01 \leq \rho_{0} \leq 0.04$ under the form of compact objects. This range is of the order of the dark matter densities estimated in our own and other galaxies from rotation curves.

\section{Future prospects}

We propose the southern blazar PKS 0537-441 as a good candidate in a search for extragalactic MACHOs. Owing to the usual superluminal motions in blazars, the optical depth and the number of events will not significantly depend on the velocity distribution of the lenses, and due to the excellent source-lens-observer alignment in the case of PKS 0537-441 there will be no dependence on peculiarities in the structure and shape of the dark halo. Detailed computations (Surpi et al. 1995) show that if the total range of lens' masses is $10^{-4} M_{\odot}-1 M_{\odot}$, then 49 events are expected over one month of continuous monitoring of the blazar at optical frequencies. The time scales goes from 30 minutes to 3 days, in case of a superluminal motion with $3 c \leq v_{\text {app }} \leq 4 c$ in the blazar.

\section{References}

Gopal-Krishna, Subramanian, K., 1991, Nature, 349, 766

Romero, G.E., Combi, J.A., Colomb, F.R., 1994, A\&A, 288, 731

Romero, G.E., Surpi, G., Vucetich, H., 1995, A\&A, in press

Stickel, M., Fried, J.W., Kühr, H., 1988, A\&A, 206, L30

Surpi, G., Romero, G.E., Vucetich, H., 1995, in preparation 